\title{
Numerical Investigation of Non-Newtonian Flow and Heat Transfer Characteristics in Rectangular Tubes with Protrusions
}

\author{
Yonghui Xie, Zheyuan Zhang, Zhongyang Shen, and Di Zhang \\ School of Energy and Power Engineering, Xian Jiaotong University, Xian, Shaanxi 710049, China \\ Correspondence should be addressed to Yonghui Xie; yhxie@mail.xjtu.edu.cn
}

Received 30 August 2014; Accepted 23 October 2014

Academic Editor: Haochun Zhang

Copyright ( 2015 Yonghui Xie et al. This is an open access article distributed under the Creative Commons Attribution License, which permits unrestricted use, distribution, and reproduction in any medium, provided the original work is properly cited.

\begin{abstract}
Flow characteristics and heat transfer performances in rectangular tubes with protrusions are numerically investigated in this paper. The thermal heat transfer enhancement of composite structures and flow resistance reduction of non-Newtonian fluid are taken advantage of to obtain a better thermal performance. Protrusion channels coupled with different CMC concentration solutions are studied, and the results are compared with that of smooth channels with water flow. The comprehensive influence of turbulence effects, structural effects, and secondary flow effects on the CMC's flow in protrusion tubes is extensively investigated. The results indicate that the variation of flow resistance parameters of shear-thinning power-law fluid often shows a nonmonotonic trend, which is different from that of water. It can be concluded that protrusion structure can effectively enhance the heat transfer of CMC solution with low pressure penalty in specific cases. Moreover, for a specific protrusion structure and a fixed flow velocity, there exists an optimal solution concentration showing the best thermal performance.
\end{abstract}

\section{Introduction}

The application of protrusion has been attracting the attention of many researchers due to its advantages for friction factors and enhancement for heat transfer. Mahmood et al. [1] studied the flow characteristics in rectangle tube with protrusions structures. They found that protrusions can strengthen the vortex, secondary flow, and unsteadiness of the fluid. Then Moon et al. [2] investigated the phenomenon of flow and heat exchange in narrow channel arrayed with dimples and protrusions. It was reported that the region of enhancing heat transfer was focused on the windward side, and, with the increased Reynolds number, coefficients of both friction and heat transfer are decreased. Borisov et al. [3] studied the characteristics of flow and heat transfer in similar channels as Moon's, and the average heat transfer coefficient and friction coefficient were obtained. They compared the flow in tubes arrayed with dimples, tubes arrayed with both dimples and protrusions, and traditional sine wave surface channels, finding that protrusions can enhance heat transfer, but it also results in extra pressure loss, which is due to the destruction of vortex structure produced by dimples. In addition, heat transfer coefficients of different channels all reach the maximum near the transition Reynolds.

Simultaneously several researchers have investigated non-Newtonian flow characteristics and its heat exchange phenomenon. Arhuoma et al. [4] studied the viscosity of water-in-oil emulsion in porous media, finding that when the concentration changed from $6.78 \%$ to $33.48 \%$, the solution showed shear-thinning features and belonged to a typical power-law fluid. They concluded that the degree of droplet size distribution had little influence on emulsion viscosity, and they reported the prediction correlations of the emulsion viscosity. Srisamran and Devahastin [5] investigated the flow and mixing behavior of steady laminar confined impinging streams of shear-thinning fluids, which was compared with that of Newtonian impinging streams. Eesa and Barigou [6] using a validated CFD model showed that a transverse vibration motion on a steady laminar flow could generate sufficient chaotic fluid motion which leads to intense radial mixing. As a result, the heat transfer is strengthened and a nearuniform radial temperature field with a substantial heating of 


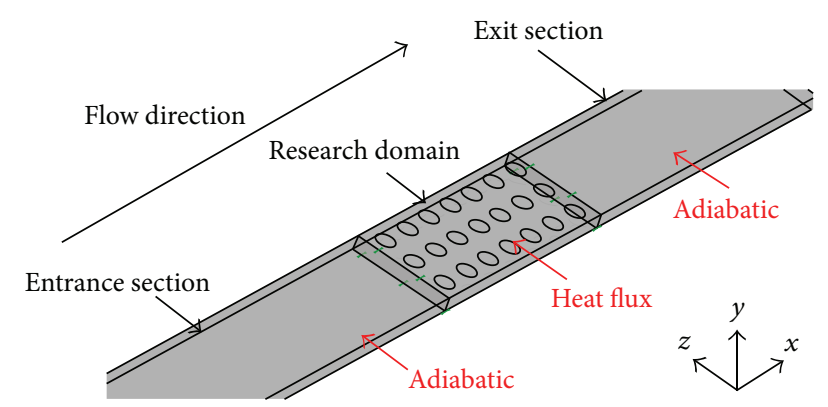

FIGURE 1: Schematic diagram of boundary conditions.

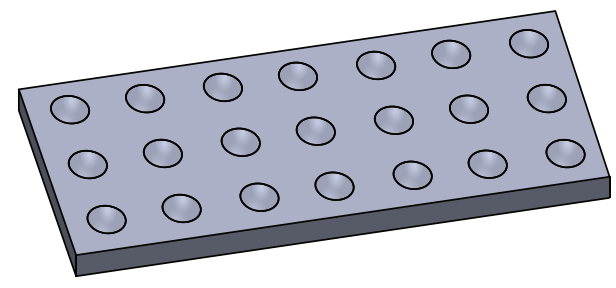

FIgURE 2: Protrusion arrangement in the research domain.

the inner flow is achieved. Pimenta and Campos [7] carried out an experimental work to obtain, for Newtonian and non-Newtonian fluids, heat transfer coefficients, at constant wall temperature, in fully developed laminar flow inside a helical coil. Hojjat et al. [8] experimentally studied the forced convective heat transfer of three kinds of nanofluids flowing in a uniformly heated circular tube under turbulent flow conditions.

Although much research has been devoted to protrusion structure and non-Newtonian fluid, respectively, little research has been done on the combination of these two parts. In this study, a numerical investigation is done to characterize the flow and heat transfer of CMC solution in rectangle tubes arrayed with protrusion structures, in order to make use of both advantages of composite structure and nonNewtonian fluid. The purpose of the present paper is to obtain a new cooling technique for industrial equipment, which can effectively enhance heat transfer with little pressure penalty in order to improve the efficiency and stability of the equipment operation.

\section{Numerical Method}

2.1. Computational Geometry and Boundary Conditions. The schematic diagram of the protrusion roughened rectangular channel used in this study is shown in Figure 1. The coordinates $x, y$, and $z$ represent the streamwise, normal, and spanwise direction, respectively. The channel is roughened with protrusions on one wall of the research section in an inline arrangement. The protrusion arrangement in the research section is shown in Figure 2. Fully developed periodic velocity

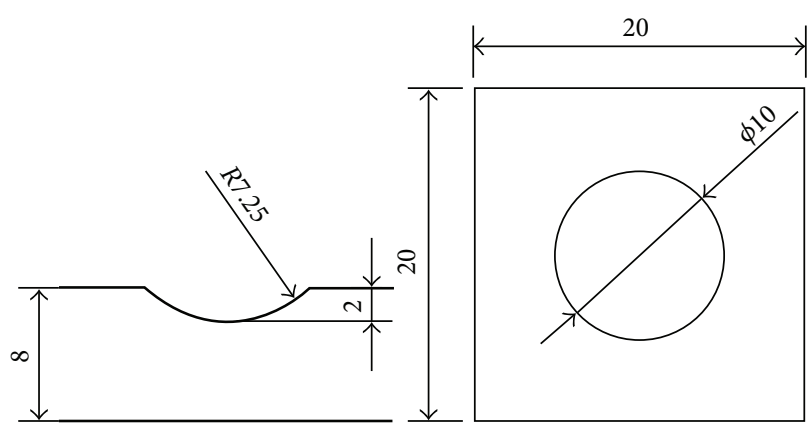

FIGURE 3: Geometry size of the protrusion unit.

and temperature can be obtained after certain streamwise rows of protrusions. Under this condition, 7 rows of protrusion structures are applied in the computational domain.

CMC solutions with three kinds of concentrations (100 ppm/500 ppm/2000 ppm) and water are employed for comparison in the present study. The baseline is the case of water flowing in smooth rectangular channel. The following boundary conditions are specified: (a) A uniform heat flux of $q=5 \times 10^{5} \mathrm{~W} \cdot \mathrm{m}^{-2}$ and no-slip boundary condition are applied at the four external surfaces of the research section. (b) The relative pressure is $1 \times 10^{5} \mathrm{~Pa}$, turbulence intensity is $5 \%$, the static temperature at inlet is $300 \mathrm{~K}$, and the static pressure at outlet is $0 \mathrm{~Pa}$. As shown in Figure 1, the research section is placed between entrance section and exit section, of which the length is more than 30 times the height, in order to ensure that the solutions enter the research section with a fully developed velocity and exit the section without the influence of non-Newtonian extrusion swelling effect.

The rectangular channel is $8 \mathrm{~mm} \times 60 \mathrm{~mm}$ in cross section. The length of the research section is $140 \mathrm{~mm}$. The detailed parameters of the protrusion unit are shown in Figure 3.

The protrusion is a sector of a sphere with radius $R=$ $7.25 \mathrm{~mm}$. The depth and diameter of the protrusion unit are, respectively, $2 \mathrm{~mm}$ and $10 \mathrm{~mm}$. The streamwise and spanwise pitch of protrusions is $20 \mathrm{~mm}$.

2.2. Solution Method. The assumption that the flow is steady, incompressible and turbulent flow. Constant fluent properties is adopted for modeling the flow characteristics and heat transfer in the protrusion channel in this paper. The incompressible steady Navier-Stokes equation is solved using the finite-volume method based solver CFX and the SST turbulent model coupled with gamma-theta transition model which is adopted in the investigation. The computations are considered to be converged when the residues for continuity, energy, velocities, and the area-averaged wall temperature are less than $1 \times 10^{-6}, 1 \times 10^{-7}, 1 \times 10^{-3}$, and $1 \times 10^{-6}$, respectively. The flow velocity in the present paper ranges from $1.289 \mathrm{~m} \cdot \mathrm{s}^{-1}$ to $3.221 \mathrm{~m} \cdot \mathrm{s}^{-1}$, and the corresponding Newtonian Reynolds number ranges from 20000 to 50000 , which is different with that of non-Newtonian fluids. 
TABLE 1: Grid independency study.

\begin{tabular}{|c|c|c|c|c|c|}
\hline Case & Total nodes & $\mathrm{Nu}$ & Difference \% & $f$ & Difference \% \\
\hline Mesh 1 & 1599456 & 136.609 & 3.31 & 0.012503 & 0.45 \\
\hline Mesh 2 & 2432201 & 139.166 & 1.50 & 0.012527 & 0.25 \\
\hline Mesh 3 & 3817146 & 140.310 & 0.69 & 0.012548 & 0.09 \\
\hline Mesh 4 & 5579691 & 141.284 & Reference & 0.012559 & Reference \\
\hline
\end{tabular}

2.3. Data Reduction. The Reynolds number of non-Newtonian fluids is described by [9]

$$
\operatorname{Re}^{\prime}=\frac{D^{n} v^{2 n-1} \rho}{8^{n-1} K}\left(\frac{4 n}{3 n+1}\right)^{n},
$$

where $v$ is the inlet velocity, $\rho$ is the density, $K$ is the consistency coefficient, and $n$ is the flow behavior index. The hydraulic diameter $D$ is given by

$$
D=\frac{4 A}{P} .
$$

The Reynolds number of Newtonian fluids is defined by

$$
\operatorname{Re}=\frac{\rho v D}{\mu},
$$

where $\mu$ is the viscosity coefficient. by

The local Nusselt number in the present study is defined

$$
\mathrm{Nu}_{x}=\frac{h_{x} D}{\lambda}
$$

where $\lambda$ is the thermal conductivity of solutions. The heat transfer coefficient $h_{x}$ is defined as

$$
h_{x}=\frac{q}{\Delta T_{x}},
$$

where $q$ represents the heat flux and $\Delta T_{x}$ is the mean temperature difference between the wall and the fluids which can be obtained as follows:

$$
\Delta T_{x}=T_{w, x}-T_{x},
$$

where $T_{w, x}$ and $T_{x}$ are the local temperature of wall and fluids, respectively.

The average Nusselt number for the channel can be calculated by

$$
\mathrm{Nu}=\frac{\left(\int \mathrm{Nu}_{x} d S\right)}{S} .
$$

The Fanning friction factor, $f$, is defined as

$$
f=-\frac{(\Delta P / L) D}{2 \rho v^{2}},
$$

where $\Delta P$ is the pressure drop and $L$ is the streamwise length of the research section.

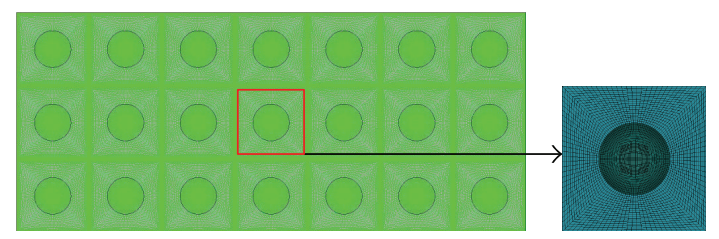

FIGURE 4: Schematic diagram of mesh on protrusion surface.

The friction loss, $C_{f}$, is given by

$$
C_{f}=\frac{X_{\text {wall }}}{(1 / 2) \rho v^{2}},
$$

where $X_{\text {wall }}$ is the wall shear stress.

The form loss can be obtained as follows:

$$
C_{p}=f-C_{f} .
$$

The thermal performance is

$$
\mathrm{TP}=\left(\frac{\mathrm{Nu}}{\mathrm{Nu}_{0}}\right) \cdot\left(\frac{f}{f_{0}}\right)^{-1 / 3} .
$$

The baseline Fanning friction factor, $f_{0}$, and Nusselt number, $\mathrm{Nu}_{0}$, for the case of water flowing in the smooth rectangular channel, are used to normalize the Fanning friction factor and Nusselt number of CMC solutions flowing in the protrusion channel.

The baseline Fanning friction factor $f_{0}$ and Nusselt number $\mathrm{Nu}_{0}$ are given as

$$
\begin{gathered}
f_{0}=\frac{0.3164}{\mathrm{Re}^{0.25}}, \\
\mathrm{Nu}_{0}=0.023 \mathrm{Re}^{0.8} \operatorname{Pr}^{n},
\end{gathered}
$$

where $P r$ is the Planck number.

2.4. Grid Independence Study. In this paper O-grids are used for the protrusion region, as shown in Figure 4, and an $\mathrm{H}$ type grid is generated for the flow core. The mesh around the protrusion region is refined. Before the numerical simulation, the grid independency study is carried out and four different grid systems are investigated. Water is used as flow medium, and the inlet velocity is set as $1.289 \mathrm{~m} \cdot \mathrm{s}^{-1}$.

Table 1 summarizes the comparisons. The $\mathrm{Nu}$ and $f$ change by $0.69 \%$ and $0.09 \%$ from mesh 3 to mesh 4 , respectively. Hence, mesh 3 is used. 
TABLE 2: Flow parameters of aqueous CMC solutions [10].

\begin{tabular}{lcc}
\hline Concentration/ppm & $n$ & $\mathrm{~K} / \mathrm{kg} \cdot \mathrm{s}^{n-2} \cdot \mathrm{m}^{-1}$ \\
\hline 100 & 0.9512 & 0.00383 \\
500 & 0.8229 & 0.00849 \\
2000 & 0.7051 & 0.02792 \\
\hline
\end{tabular}

TABLE 3: Physical properties of CMC solutions used in the simulation [11].

\begin{tabular}{lc}
\hline Property & Value \\
\hline Density $(\rho)$ & $1000 \mathrm{~kg} \cdot \mathrm{m}^{-3}$ \\
Specific heat $\left(C_{P}\right)$ & $4100 \mathrm{~J} \cdot \mathrm{kg}^{-1} \cdot \mathrm{K}^{-1}$ \\
Thermal conductivity $(\lambda)$ & $0.7 \mathrm{~W} \cdot \mathrm{m}^{-1} \cdot \mathrm{K}^{-1}$ \\
\hline
\end{tabular}

2.5. Physical Properties. In this study, water and CMC (carboxyl methyl cellulose) solutions are used as typical Newtonian and shear-thinning fluids, respectively. The CMC solutions exhibit pseudoplastic (shear-thinning) feature. Table 2 gives the values of the flow behavior index and the consistency coefficient of CMC solutions at different concentrations, and the physical properties of the CMC solutions are listed in Table 3 . The temperature difference of the inlet and outlet is lower than $5^{\circ} \mathrm{C}$ and the potential effects of the temperature-dependent thermophysical properties can be decoupled. Hence the properties of fluids are assumed to be constant in this study.

\section{Results and Discussion}

3.1. Flow Characteristics of the Protrusions. In the present paper, the center plane in the flow direction (Figure 5) is chosen for the investigation. In order to study the flow of different CMC solutions, the distribution of streamline and turbulence intermittency $\gamma$ for the protrusion regions at $v=$ $1.289 \mathrm{~m} \cdot \mathrm{s}^{-1}$ is shown in Figure 6.

Through an examination of the case at $v=1.289 \mathrm{~m} \cdot \mathrm{s}^{-1}$, the streamline distributions of different CMC concentration solutions are nearly the same. On the protrusion side, separation is identified downstream of the protrusion. At the same time, a smaller separation zone can be observed as the flow slows down before it impinges on the front of the protrusion.

The turbulence intermittency $\gamma$ can be used as the criteria of flow state at fixed point: at $\gamma=0$, the flow remains laminar, and, at $\gamma \geq 1$, the flow is fully developed turbulent. In the range of $0<\gamma<1$, the flow is in transition condition. As shown in Figure 6, for a specific kind of solution, the biggest laminar area appears in the first protrusion region and decreases along the flow direction. Meanwhile, for the specific position of protrusion, the laminar area increases as the solution concentration increases. In fact, at $v=1.289 \mathrm{~m} \cdot \mathrm{s}^{-1}$, the flow of water in the protrusion channel has reached fully developed condition and remains invariable as the velocity increases. However, there is also a certain amount of laminar area existing in the flow of CMC solutions. It can be concluded that it is harder for the CMC solution flow

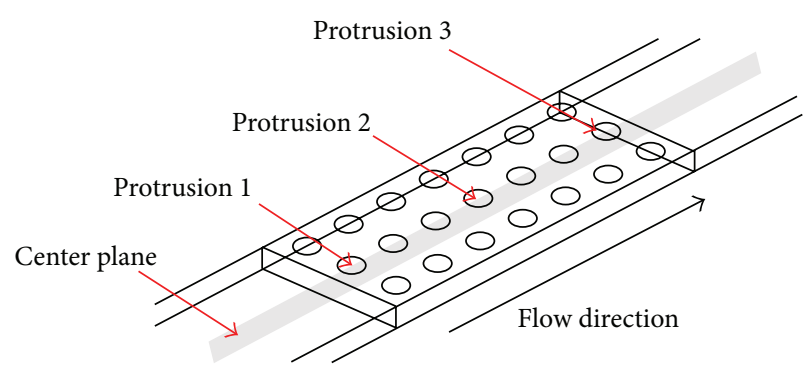

FIGURE 5: Schematic diagram of the center plane in the flow direction.

with higher concentration to reach fully developed turbulent condition at the same velocity due to its higher viscosity.

It is worth pointing out that the turbulence intermittency instead of Reynolds number is used to estimate flow state, because it can illustrate the transient process more clearly and the transition Reynolds numbers of CMC solutions are unknown.

In order to understand the influence of different flow conditions, two typical protrusion regions are chosen to study, which are shown by the blocks in Figure 7.

Figure 8 shows the distribution of streamline and temperature in two typical protrusion regions at $v=2.255 \mathrm{~m} \cdot \mathrm{s}^{-1}$ and $v=3.221 \mathrm{~m} \cdot \mathrm{s}^{-1}$. As observed the fluids imping on the front edge of protrusions result in small recirculation zones. Then the fluids detour around the side edge of protrusions, leading to strong perturbation and high flow velocity. Besides, the flow separation taking place in the trailing edges produces a large separation zone. For the protrusions in symmetrical location, it is worth pointing out that a dominant feature of streamline distribution is that there are two focuses symmetrical about the center plane of them. The two focuses are considered to be the feet of the horseshoe vortex structure. In the region near the flow impingement, the temperature is relatively lower than that in other regions, and in the separation zone the temperature is relatively higher.

Through an examination of the flow structures of all the cases, the differences in concentration solution and velocity lead to the variation in size and location of recirculation zones and separation zones. Taking the case of $v=2.255 \mathrm{~m} \cdot \mathrm{s}^{-1}$ as an example, as concentration increases, the recirculation zone at the front edges of upstream protrusions increases, while the separation zone at the trailing edges of midstream protrusions decreases, as presented in Figure 8.

3.2. Characteristics of Secondary Flow. In order to study the secondary flow in the tube and the interaction between protrusion perturbation and non-Newtonian secondary flow effect, two cross sections perpendicular to the flow direction are chosen, as shown in Figure 9.

For cross section 1, the flow structures in different protrusion regions are similar and the streamline distribution is symmetrical, as shown in Figure 10. As a result, the flow structure in the mid-protrusion region is chosen for the following study. The streamline distribution of different solutions in 


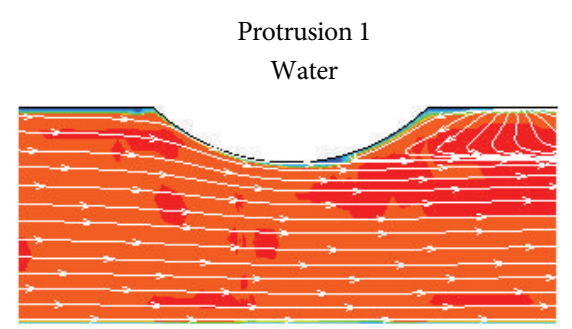

CMC100

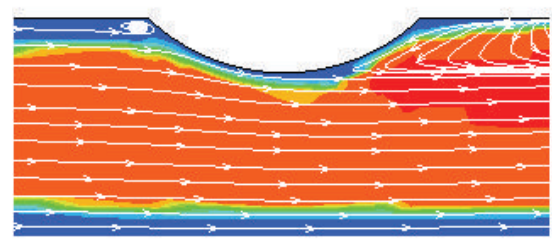

CMC500

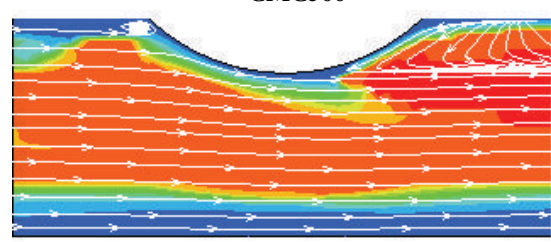

CMC2000
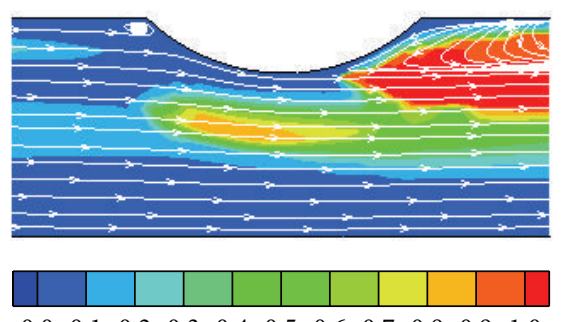

Protrusion 2

Water

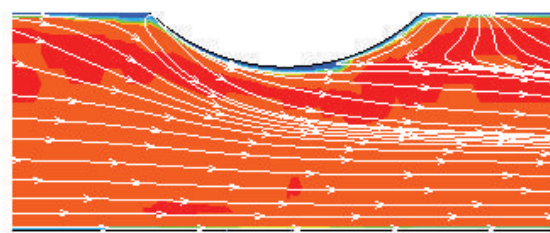

CMC100

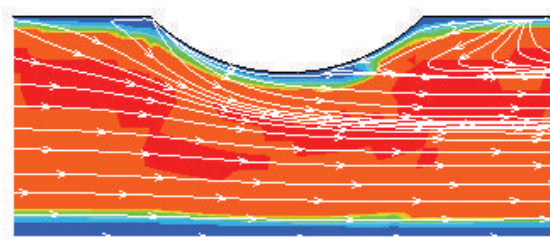

CMC500

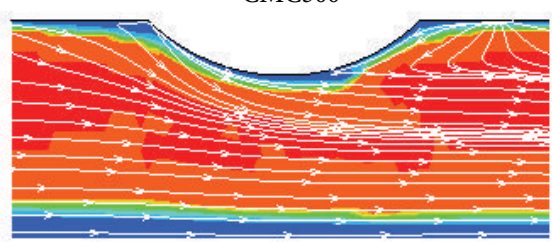

CMC2000
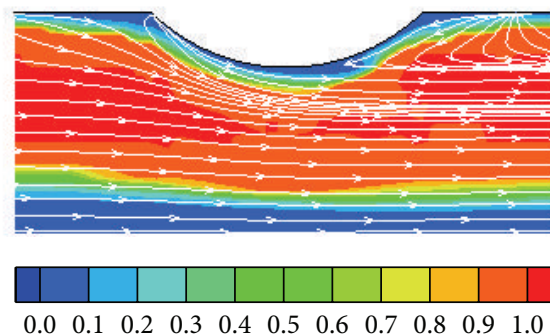

Protrusion 3

Water

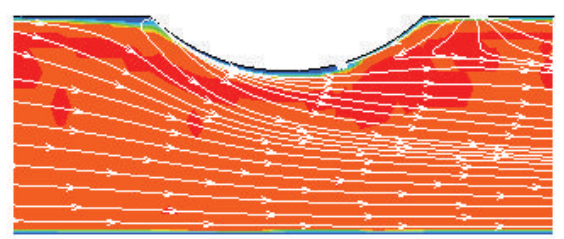

$\mathrm{CMC} 100$

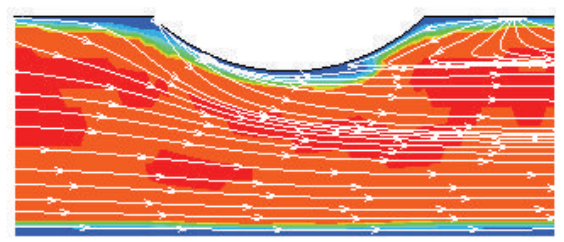

CMC500

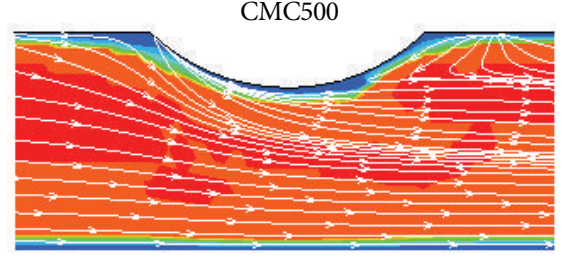

CMC2000

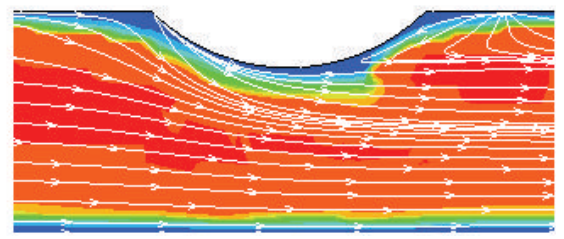

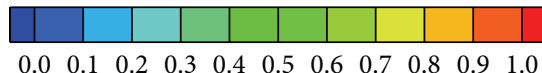

FIGURE 6: Distribution of streamline and turbulence intermittency.

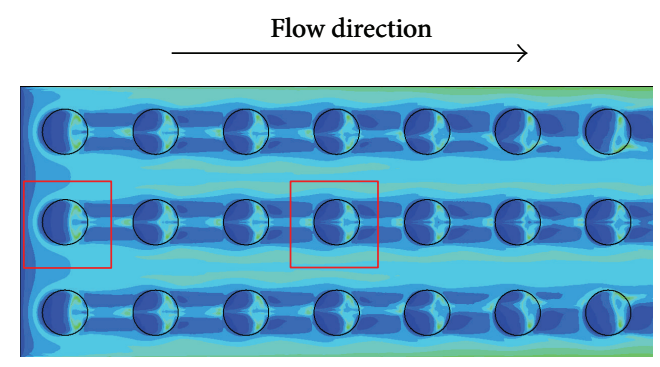

FIGURE 7: Schematic diagram of the protrusion unit.

mid-protrusion region at $v=2.255 \mathrm{~m} \cdot \mathrm{s}^{-1}$ is shown in Figure 11. As observed, the streamline distribution in protrusion region shows a dual symmetrical vortex structure. The first one is under protrusions and the second one which shows ellipse shape is at the protrusion edge side. The two pairs of vortices rotate in the opposite direction. The influence of concentration is mainly reflected by the size and the shape of the second vortex structures. At $v=2.255 \mathrm{~m} \cdot \mathrm{s}^{-1}$ the location of the second vortex of higher concentration solution is more closed to the upper surface and the shape of the second vortex is more oblate. This phenomenon is due to the combined effect of non-Newtonian secondary flow and perturbation from protrusions.

Then the present section chose cross section 2 to illustrate the single effect of secondary flow. The streamline distributions of different solution at $v=1.289 \mathrm{~m} \cdot \mathrm{s}^{-1}$ and $v=$ $3.221 \mathrm{~m} \cdot \mathrm{s}^{-1}$ are shown in Figure 12. As observed in the smooth rectangular channel, the flow can be divided into two parts. One is the flow in boundary region, which is related to turbulent effect and shown in blocks. The other is the secondary flow region which is related to viscosity of non-Newtonian fluid. The boundary region area increases with the increasing inlet velocity and the secondary flow region area increases with the increasing concentration of solutions. More specifically, in the case of $v=3.221 \mathrm{~m} \cdot \mathrm{s}^{-1}$, the boundary region of water and CMC100 is obviously bigger than that at $v=1.289 \mathrm{~m} \cdot \mathrm{s}^{-1}$, and a boundary region appears in the flow of CMC500 and CMC2000, which does not exist at $v=1.289 \mathrm{~m} \cdot \mathrm{s}^{-1}$. The difference in the streamline distribution between the two cross sections indicates that, in the present situation, the effect of protrusion dominates the flow characteristics and the variation in solution viscosity just 


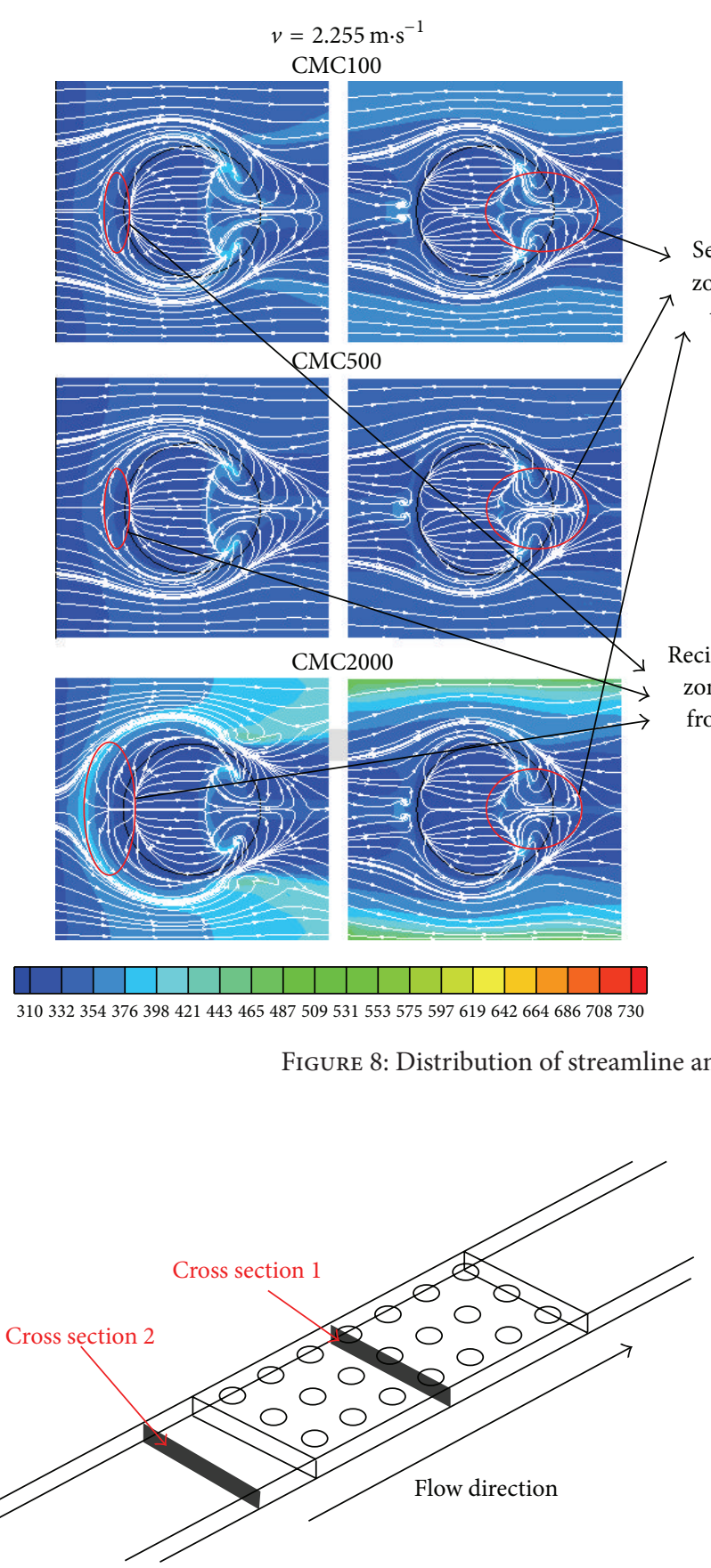

FIgURE 9: Schematic diagram of the research cross sections.

changes the flow distribution in a small part of the research regions.

According to the results above, the flow in rectangular tubes is influenced by the combined effect of turbulence effect, secondary flow effect, and the perturbation of protrusions, which results in the variation in dimensional flow characteristics for different cases.

3.3. Heat Transfer Characteristics. The distribution of local Nusselt number of different CMC concentration solutions at $v=2.255 \mathrm{~m} \cdot \mathrm{s}^{-1}$ on protrusion surface is shown in Figure 13.
As observed the flows of CMC solution in tubes arrayed with protrusion structures all show high Nusselt number at the front edge of protrusions and low Nusselt number at the trailing edge of protrusions, which is because the flow impingement at the front edge strengthens the perturbation and the flow separation at the trailing edge weakens the exchange of mass and energy. This is in accordance with the analysis of flow characteristics above.

3.4. Flow Resistance Characteristics. Figure 14 describes the variation of flow resistance parameters at different CMC concentrations as the inlet velocity increases from $1.289 \mathrm{~m} \cdot \mathrm{s}^{-1}$ to $3.221 \mathrm{~m} \cdot \mathrm{s}^{-1}$. Four flow resistance parameters of Fanning friction coefficient, normalized friction coefficient, friction loss, and form loss are selected. As observed the flow resistance parameters of $\mathrm{CMC}$ solution in protrusion tubes often show nonmonotonous variation tendency as velocity increases. The variations of flow resistance parameters of different CMC concentrations solution are also different in the range of present study. To be specific, the flow resistance parameters of CMC100 and CMC500 increase at first and then decrease, while the tendency for CMC2000 decreases at first and then increases. The protrusion structures destroy the development of the boundary layer, thus producing the form loss due to the adverse pressure gradient inside the separation zone. Although the shear stress in the boundary 


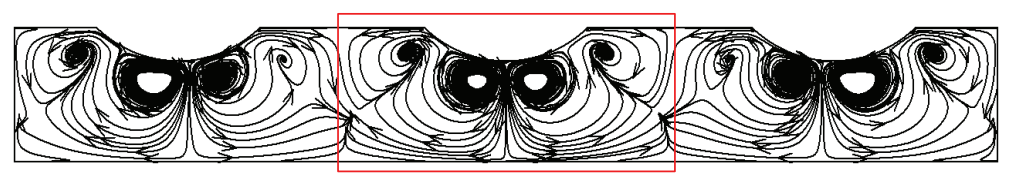

FIGURE 10: Schematic diagram of the protrusion region in the cross section 1.

Water
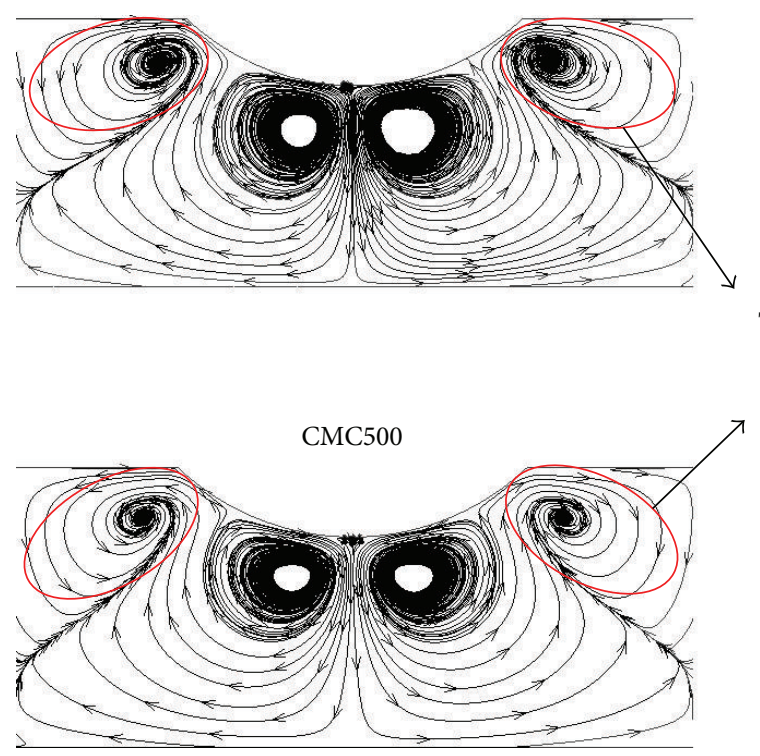

CMC100

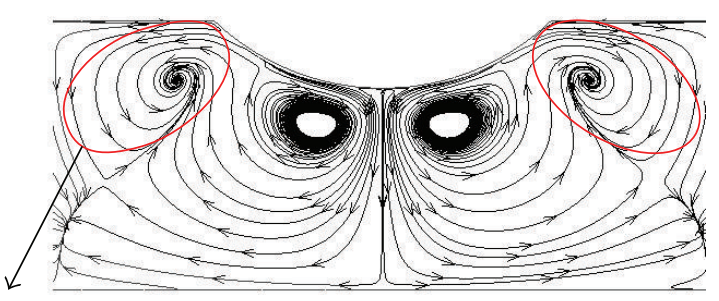

The second vortex structure

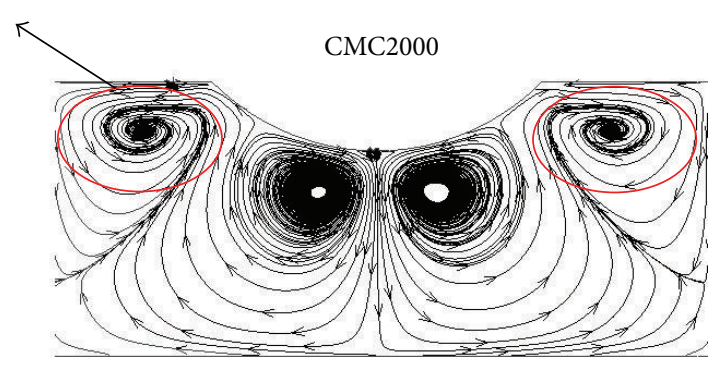

FIGURE 11: Streamline distribution of the protrusion region in the cross section 1.

layer increases, the thickness of the boundary layer decreases at the same time, which leads to the increase of friction loss. To sum up, the protrusion structure produces an extra pressure penalty. However the pressure penalty can be reduced by the effect of non-Newtonian secondary flow. For CMC2000, when the velocity is lower than approximately $2.5 \mathrm{~m} \cdot \mathrm{s}^{-1}$, the effect of the decrease in pressure penalty caused by nonNewtonian secondary flow dominates variation of the total friction. At $v>2.5 \mathrm{~m} \cdot \mathrm{s}^{-1}$, the effect of increasing the pressure penalty by protrusion structures dominates the variation of total friction coefficient. However, for CMC100 and CMC500, the domination order is opposite and the turning point is at about $v=2.0 \mathrm{~m} \cdot \mathrm{s}^{-1}$. Also, the $f / f_{0}$ values are always larger than 1 , which indicates that the effect of protrusion is stronger than that of the secondary flow. As a result, the pressure penalty of CMC solution flowing in protrusion channels is higher than that of water flowing in smooth channels.

3.5. Heat Transfer Performance. Being different from the parameters of flow characteristics, the average Nusselt number and normalized Nusselt number vary monotonously among the range of the inlet velocity studied. Compared with the flow in smooth channels, the protrusion structures can effectively strengthen the exchange of mass and energy in the boundary region, resulting in the heat transfer enhancement in channels. At the same time, the non-Newtonian secondary flow in the center regions of channels has a negative effect on the heat transfer performance. However, as shown in Figure 15 , the $\mathrm{Nu} / \mathrm{Nu}_{0}$ values are always larger than 1 , which illustrates that the effect of protrusion dominates the heat transfer characteristics and leads to the heat transfer enhancement.

3.6. Overall Thermal Performance. Figure 16 shows the overall thermal performance of different concentration solutions flowing in protrusion rectangular tubes and smooth rectangular tubes. In Figure 16 "pr" means the protrusion tubes and "sm" means smooth tubes. As observed, the overall thermal performance parameter of each combination increases with inlet velocity ranging from $1.289 \mathrm{~m} \cdot \mathrm{s}^{-1}$ to $3.221 \mathrm{~m} \cdot \mathrm{s}^{-1}$. At the same inlet velocity, the CMC500 solution in protrusion tubes shows the best thermal performance, while CMC100 solution in smooth tubes shows the worst thermal performance. However, at a low inlet velocity $\left(v<1.8 \mathrm{~m} \cdot \mathrm{s}^{-1}\right)$, the values of "CMC500 + pr" are lower than 1 in the present study, which means that the combination of protrusion structures and shear-thinning fluids improves the thermal performance only in the specific situations. In other words, the specific channel structure combined with corresponding concentration of 


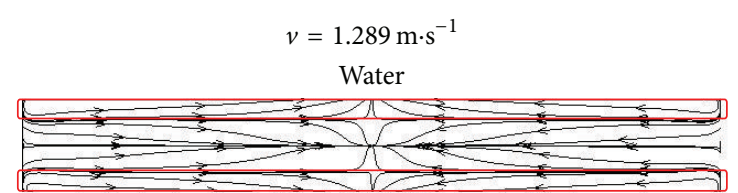

CMC100

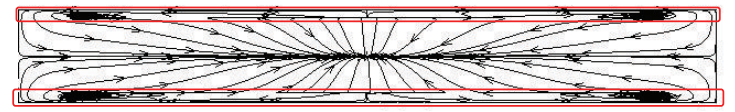

CMC500

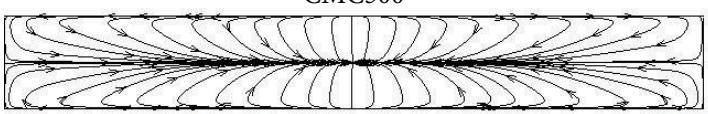

CMC2000

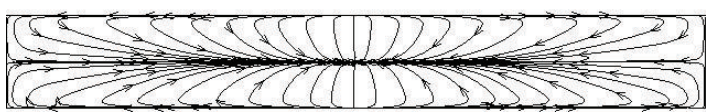

$v=3.221 \mathrm{~m} \cdot \mathrm{s}^{-1}$

Water

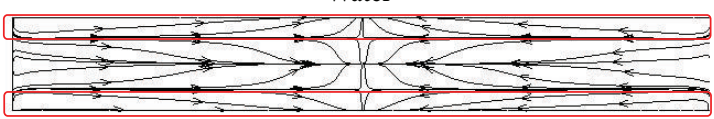

CMC100

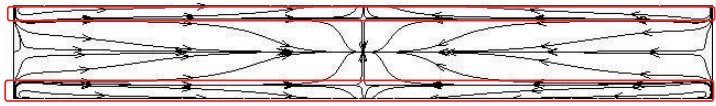

CMC500

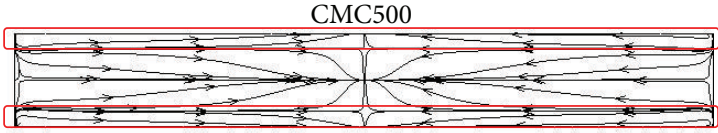

CMC2000

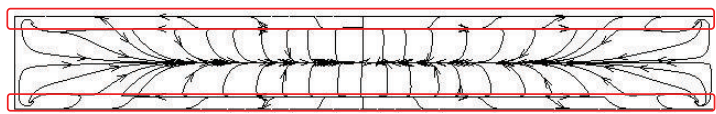

FIGURE 12: Streamline distribution of the research cross section 2.

CMC100

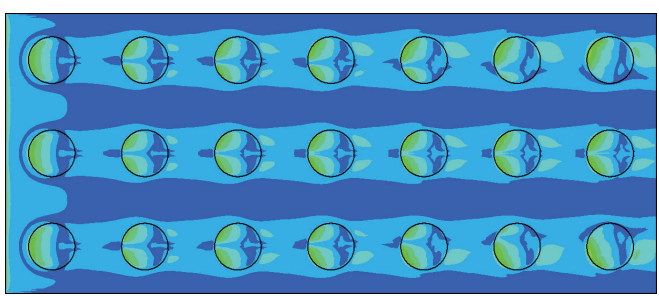

CMC500

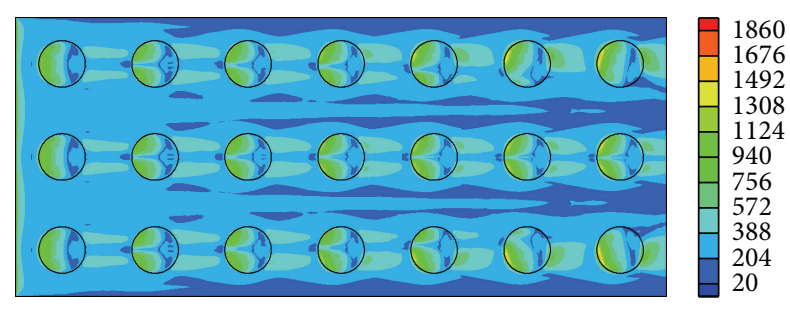

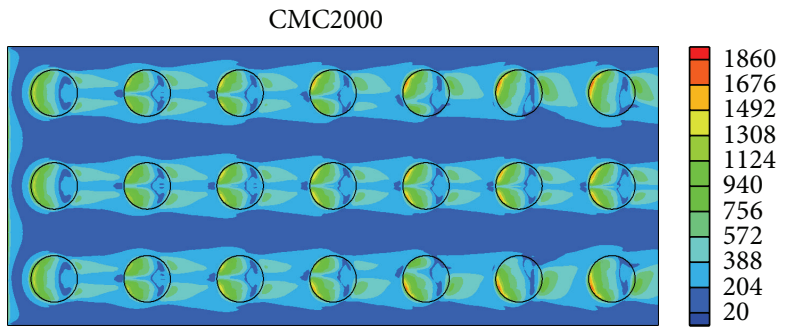

FIGURE 13: Distribution of Nusselt number on the protrusion surface.

solution and flow velocity is expected to possess an optimal thermal performance.

\section{Conclusion}

Based on the analytical and numerical investigations presented in this paper, the following conclusions can be obtained.

(1) The turbulence intermittency is adopted to estimate flow state of different CMC solutions. It can be concluded that it is harder for the CMC solution flow with higher concentration to reach fully developed turbulent condition at the same velocity due to its higher viscosity. At the same time, as observed on the protrusion side, separation is identified downstream of the protrusion; a smaller separation zone can be observed as the flow slows down before it impinges on the windward side of the protrusion.

(2) Through the observation of the flow structures on the protrusion surface, the differences in concentration solution and velocity lead to the variation in size and location of recirculation zones and separation zones, which can be attributed to the combined effect of solution viscosity and perturbation from protrusions.

(3) The flow patterns of the cross section in the protrusion channel are compared with those in the smooth channel. In the protrusion rectangular channel, the streamline distribution shows a dual symmetrical vortex structure and the influence of concentrate is mainly reflected by the size and the shape of the second vortex structures and the effect of protrusion dominates the flow characteristics and the variation 

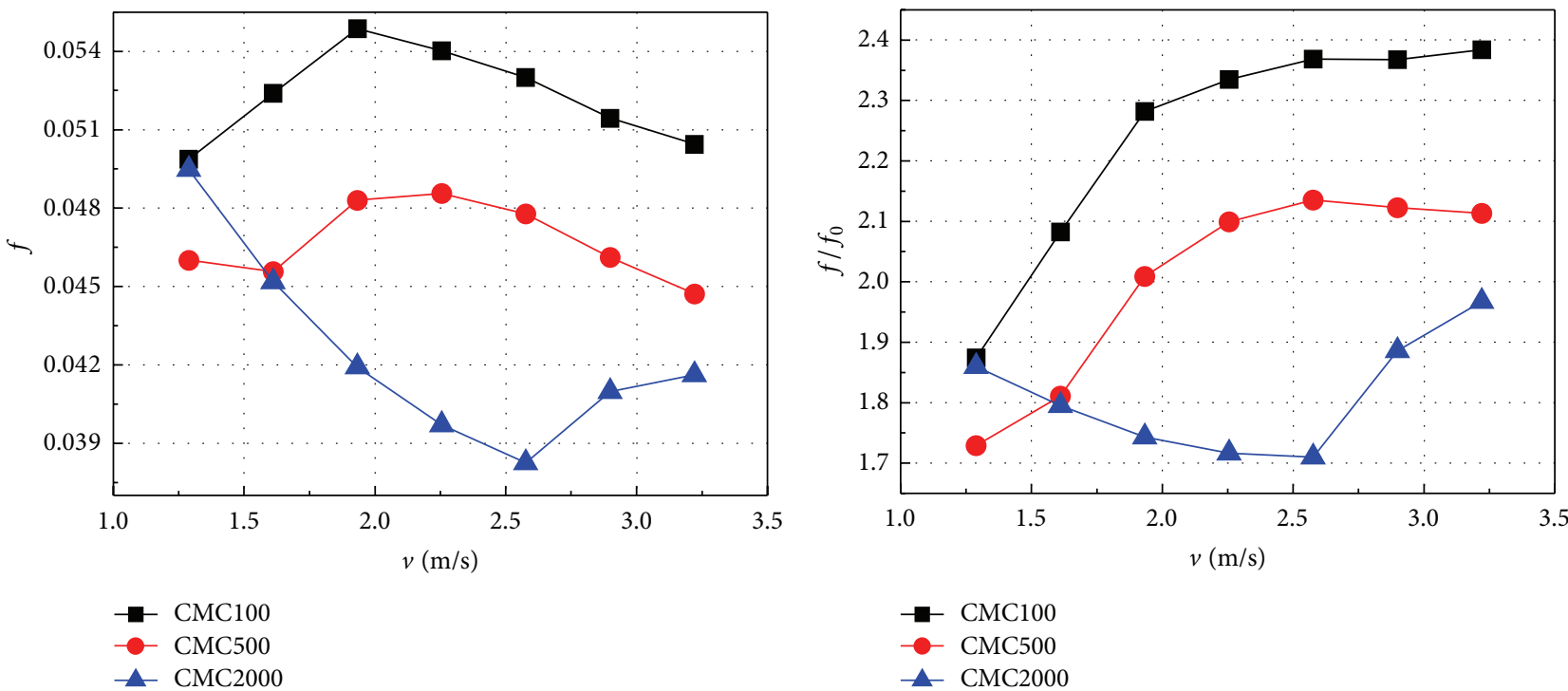

(a) Fanning friction coefficient

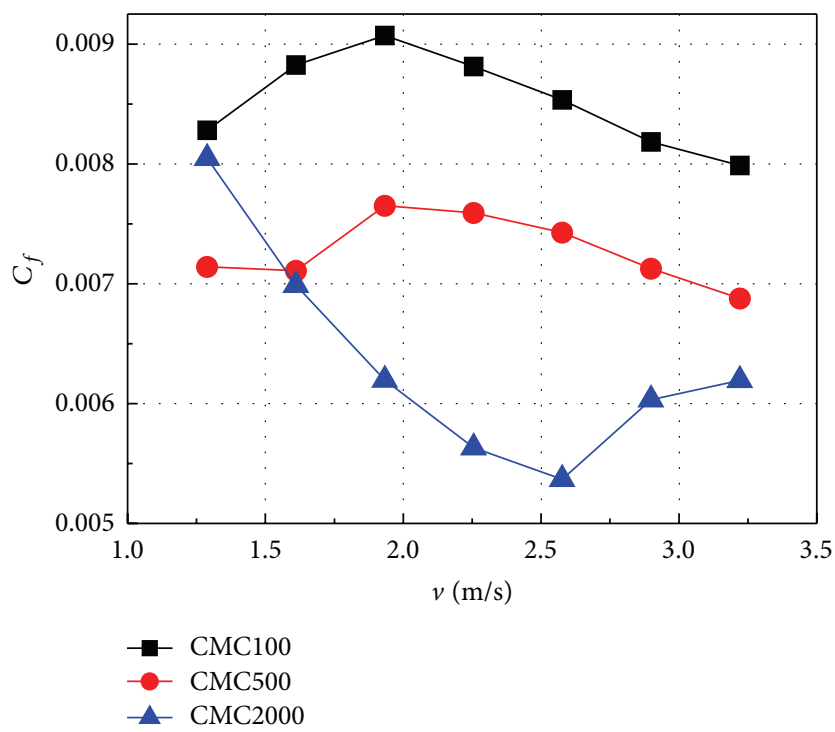

(c) Friction loss

\section{$\rightarrow-\mathrm{CMC} 100$ \\ - CMC500 \\ CMC2000}

(b) Normalized friction coefficient

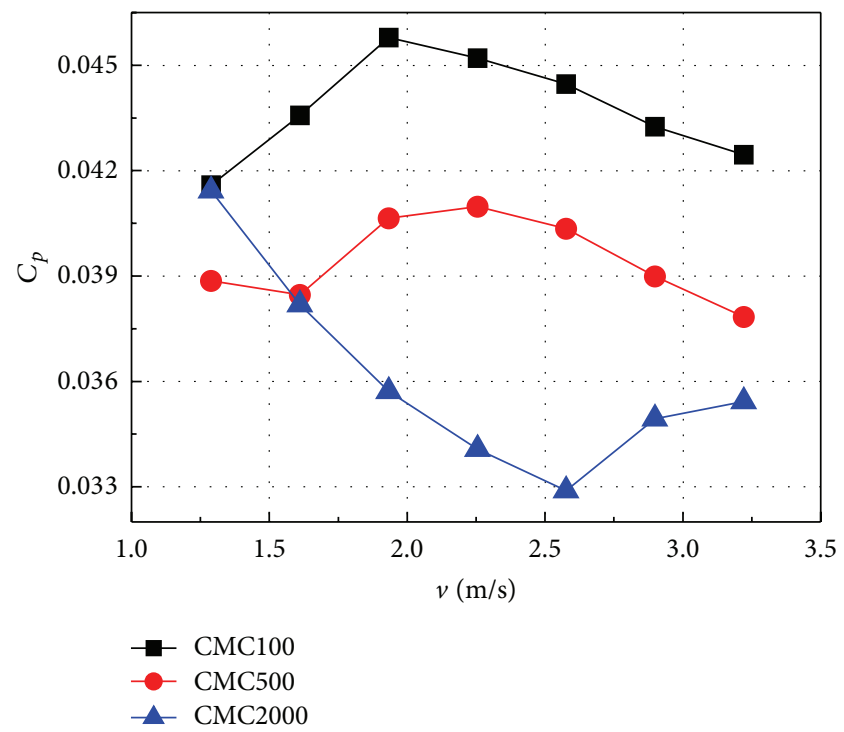

(d) Form loss

FIGURE 14: Variation of flow resistance parameters of CMC solution: (a) Fanning friction coefficient, (b) normalized friction coefficient, (c) friction loss, and (d) form loss.

in solution viscosity just changes the flow distribution in a small part of the research regions. In the smooth rectangular channel, the flow region can be divided into the boundary region and the secondary flow region. The difference in the streamline distribution between the two cross sections indicates that, in the present situation, the effect of protrusion dominates the flow characteristics.

(4) The characteristics of CMC solution flow in protrusion tubes often show nonmonotonous variation tendency as velocity increases. The flow resistance parameters of CMC100 and CMC500 increase at first and then decrease, while tendency for CMC2000 decreases at first and then increases. It is mainly due to the combined effect of turbulence effects, structural effects, and secondary flow effects. The $f / f_{0}$ values are always larger than 1 , which illustrates that the pressure penalty of CMC solution flowing in protrusion channels is higher than that of water flowing in smooth channels.

(5) The average Nusselt number and normalized Nusselt number vary monotonously among the range of the inlet velocity studied. The heat transfer can be strengthened by the protrusion structures and weakened by the effect of non-Newtonian secondary flow. The $\mathrm{Nu} / \mathrm{Nu}_{0}$ values are always larger than 1 , which illustrates that the effect of protrusion dominates the heat transfer characteristics. 

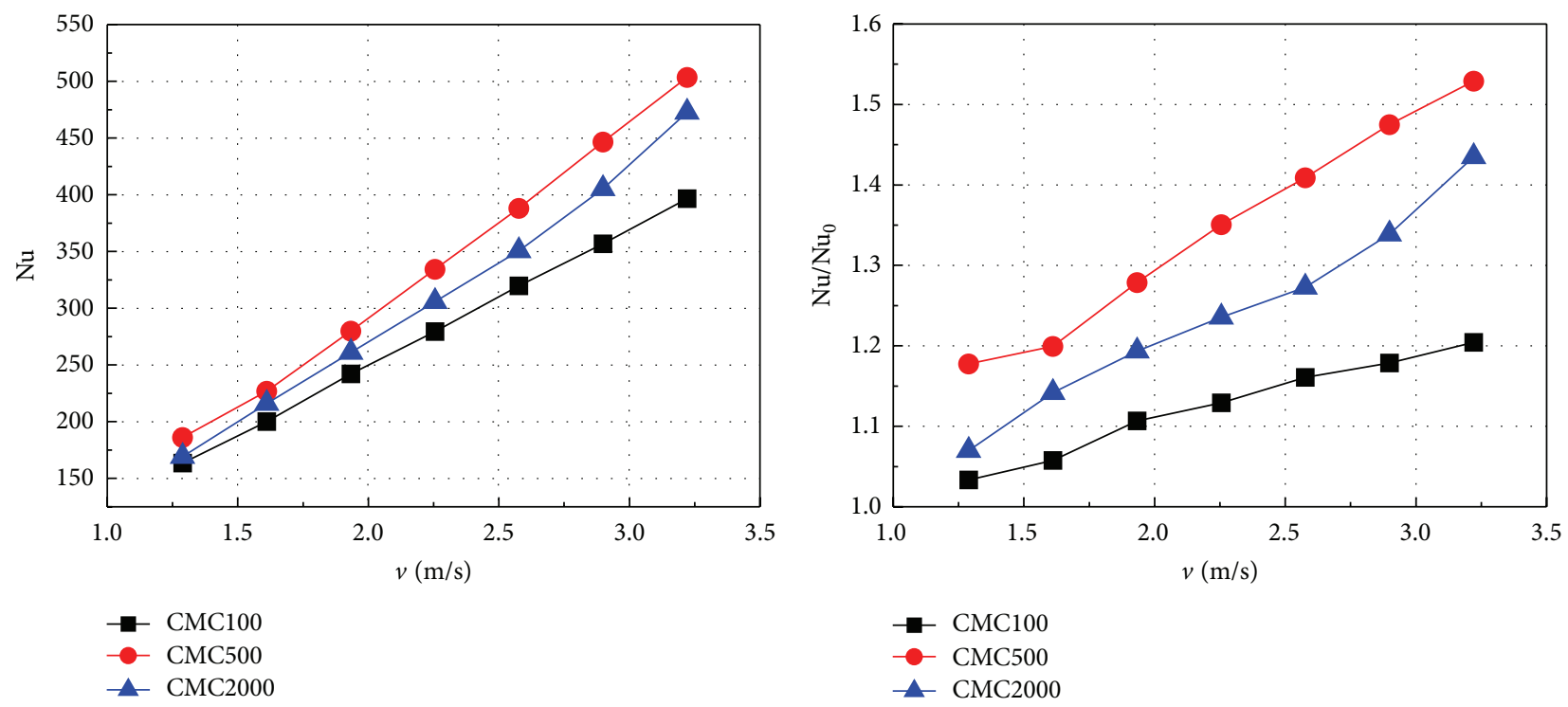

Figure 15: Variation of $\mathrm{Nu}$ and $\mathrm{Nu} / \mathrm{Nu}_{0}$ at different $\mathrm{CMC}$ concentrations.

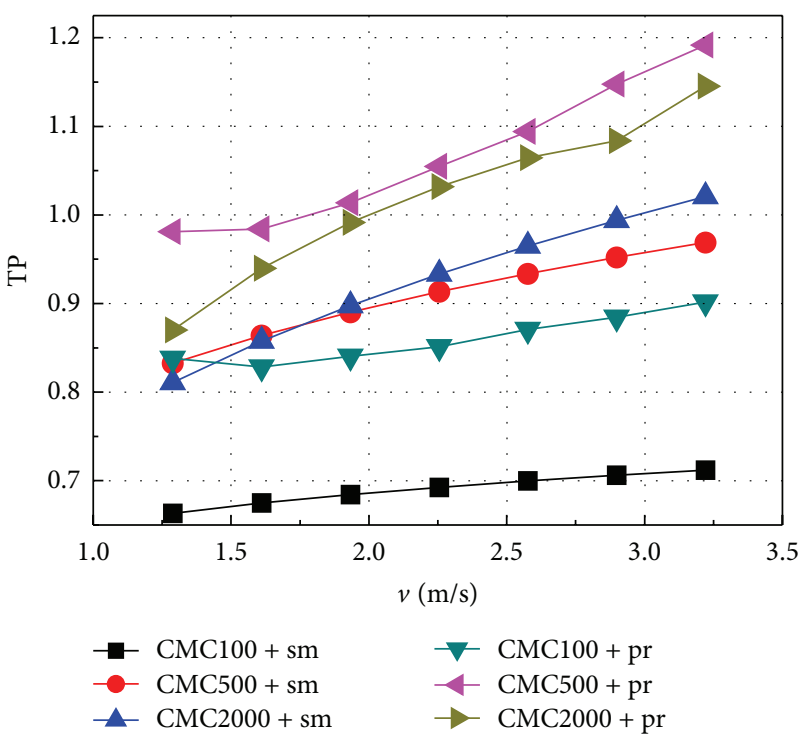

FIGURE 16: Overall thermal performance of CMC solutions.

(6) In this paper, at the same inlet velocity, the CMC500 solution in protrusion tubes shows the best thermal performance, while CMC100 solution in smooth tubes shows the worst thermal performance. It can be concluded that the specific channel structure combined with corresponding concentration of solution and flow velocity will possess optimal thermal performance.

\section{Nomenclature}

$\tau$ : Shear stress/N

$\gamma:$ Shear rate $/ \mathrm{s}^{-1}$

$V:$ Velocity vector $/ \mathrm{m} \cdot \mathrm{s}^{-1}$ p: $\quad$ Pressure $/ \mathrm{Pa}$

$f_{v}: \quad$ Body force/N

$\mu: \quad$ Viscosity coefficient $/ \mathrm{N} \cdot \mathrm{s} \cdot \mathrm{m}^{-2}$

$K: \quad$ Consistency coefficient $/ \mathrm{kg} \cdot \mathrm{s}^{n-2} \cdot \mathrm{m}^{-1}$

$n$ : $\quad$ Flow behavior index

D: $\quad$ Hydraulic diameter $/ \mathrm{m}$

A: $\quad$ Inlet cross section area $/ \mathrm{m}^{2}$

S: $\quad$ Target surface area $/ \mathrm{m}^{2}$

$P: \quad$ Wet perimeter $/ \mathrm{m}$

$\rho: \quad$ Density $/ \mathrm{kg} \cdot \mathrm{m}^{-3}$

$v: \quad$ Inlet velocity $/ \mathrm{m} \cdot \mathrm{s}^{-1}$

$q$ : $\quad$ Heat flux $/ \mathrm{W} \cdot \mathrm{m}^{-2}$

$h_{x}: \quad$ Heat transfer coefficient $/ \mathrm{W} \cdot \mathrm{m}^{-2} \cdot \mathrm{K}^{-1}$

$T_{w, x}$ : Local wall temperature/K

$T_{x}$ : $\quad$ Local fluid temperature/K

$\Delta P: \quad$ Pressure drop/Pa

TP: Overall thermal performance

$\mathrm{Nu}$ : Average Nusselt number

$\mathrm{Nu}_{0}$ : Reference Nusselt number

$\mathrm{Nu} / \mathrm{Nu}_{0}$ : Normalized Nusselt number

$f: \quad$ Fanning friction coefficient

$f_{0}$ : $\quad$ Reference Nusselt number

$f / f_{0}$ : Normalized friction coefficient

$C_{f}: \quad$ Friction loss

$C_{p}: \quad$ Form loss

Re: Newtonian Reynolds number

Re' $^{\prime} \quad$ Non-Newtonian Reynolds number

Pr: $\quad$ Planck number

L: $\quad$ Streamwise length/m

$X_{\text {wall }}: \quad$ Wall shear stress/N

$\lambda: \quad$ Thermal conductivity coefficient $/ \mathrm{W} \cdot \mathrm{m}^{-1}$. $\mathrm{K}^{-1}$

$\bar{\lambda}: \quad$ Time constant. 


\section{Conflict of Interests}

The authors declare that there is no conflict of interests regarding the publication of this paper.

\section{References}

[1] G. I. Mahmood, M. Z. Sabbagh, and P. M. Ligrani, "Heat transfer in a channel with dimples and protrusions on opposite walls," Journal of Thermophysics and Heat Transfer, vol. 15, no. 3, pp. 275-283, 2001.

[2] H. K. Moon, OT. Connell, and R. Sharma, "Heat transfer enhancement using a convex-patterned surface," in Proceedings of the ASME Turbo Expo 2002: Power for Land, Sea, and Air, pp. 887-895, American Society of Mechanical Engineers, 2002.

[3] I. Borisov, A. Khalatov, S. Kobzar, and B. Glezer, "Heat transfer and pressure losses in a narrow dimpled channel structured with spherical protrusions," in Proceedings of the ASME Turbo Expo 2006: Power for Land, Sea, and Air, pp. 191-197, American Society of Mechanical Engineers, 2006.

[4] M. Arhuoma, M. Dong, D. Yang, and R. Idem, "Determination of water-in-oil emulsion viscosity in porous media," Industrial \& Engineering Chemistry Research, vol. 48, no. 15, pp. 70927102, 2009.

[5] C. Srisamran and S. Devahastin, "Numerical simulation of flow and mixing behavior of impinging streams of shear-thinning fluids," Chemical Engineering Science, vol. 61, no. 15, pp. 48844892, 2006.

[6] M. Eesa and M. Barigou, "Enhancing radial temperature uniformity and boundary layer development in viscous Newtonian and non-Newtonian flow by transverse oscillations: a CFD study," Chemical Engineering Science, vol. 65, no. 6, pp. 21992212, 2010.

[7] T. A. Pimenta and J. B. L. M. Campos, "Heat transfer coefficients from Newtonian and non-Newtonian fluids flowing in laminar regime in a helical coil," International Journal of Heat and Mass Transfer, vol. 58, no. 1-2, pp. 676-690, 2013.

[8] M. Hojjat, S. G. Etemad, R. Bagheri, and J. Thibault, "Convective heat transfer of non-Newtonian nanofluids through a uniformly heated circular tube," International Journal of Thermal Sciences, vol. 50, no. 4, pp. 525-531, 2011.

[9] A. B. Metzner and J. C. Reed, "Flow of non-newtonian fluidscorrelation of the laminar, transition, and turbulent-flow regions," AIChE Journal, vol. 1, no. 4, pp. 434-440, 1955.

[10] H. J. Poh, K. Kumar, H. S. Chiang, and A. S. Mujumdar, "Heat transfer from a laminar impinging jet of a power law fluid," International Communications in Heat and Mass Transfer, vol. 31, no. 2, pp. 241-249, 2004.

[11] A. Kumar and M. Bhattacharya, "Transient temperature and velocity profiles in a canned non-Newtonian liquid food during sterilization in a still-cook retort," International Journal of Heat and Mass Transfer, vol. 34, no. 4-5, pp. 1083-1096, 1991. 


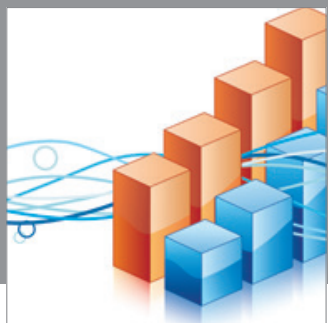

Advances in

Operations Research

mansans

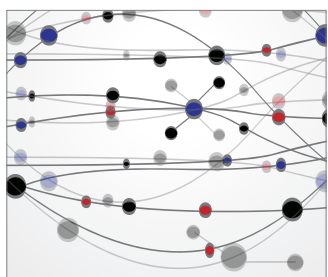

The Scientific World Journal
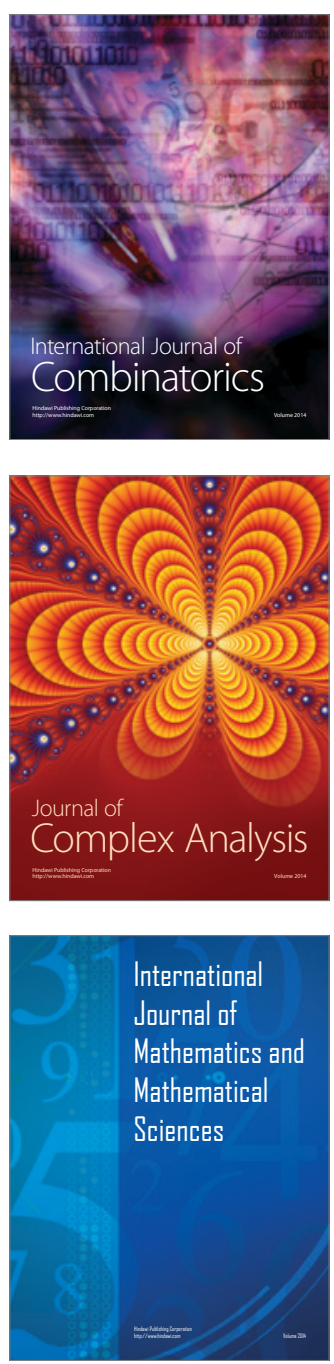
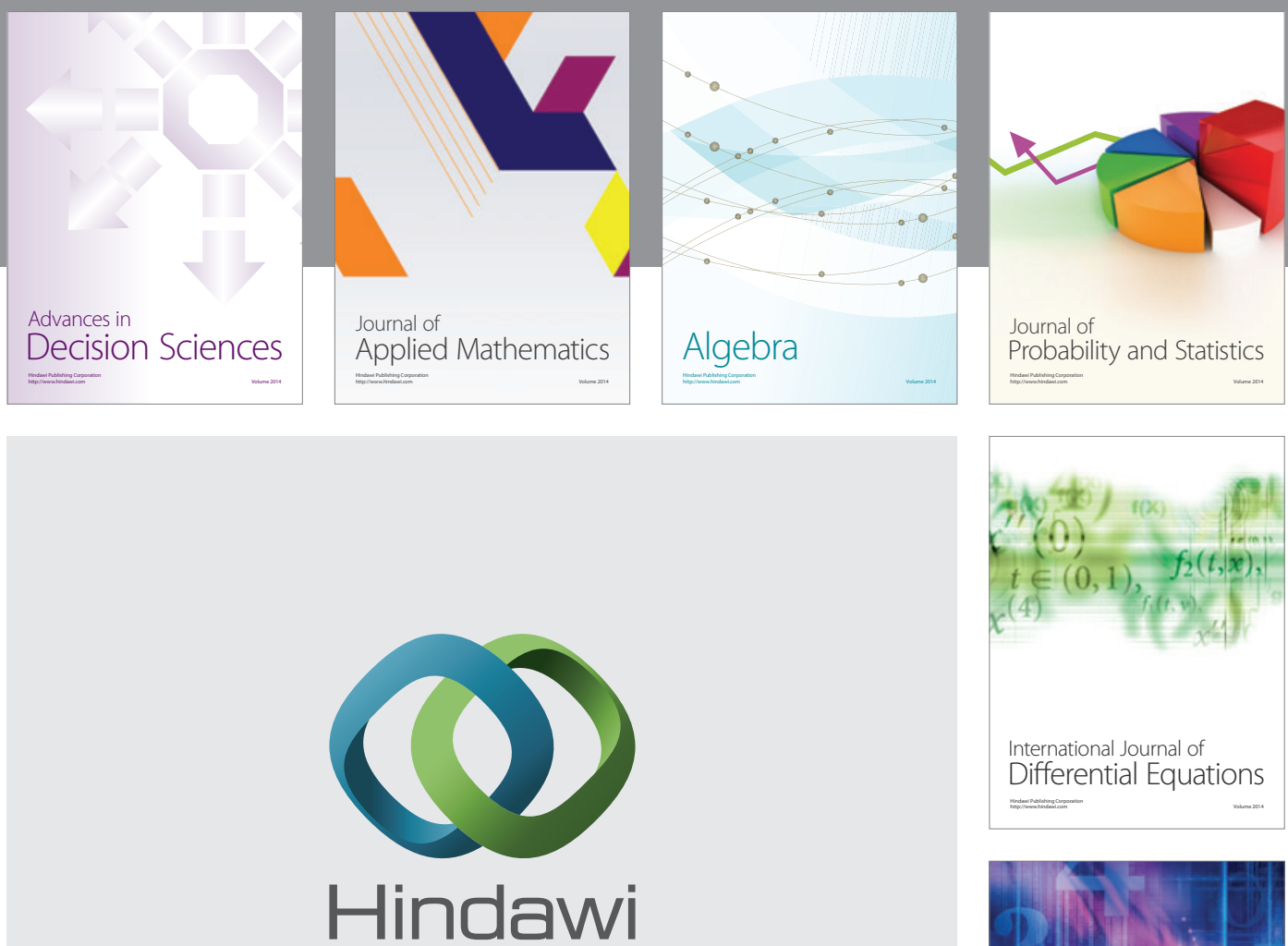

Submit your manuscripts at http://www.hindawi.com
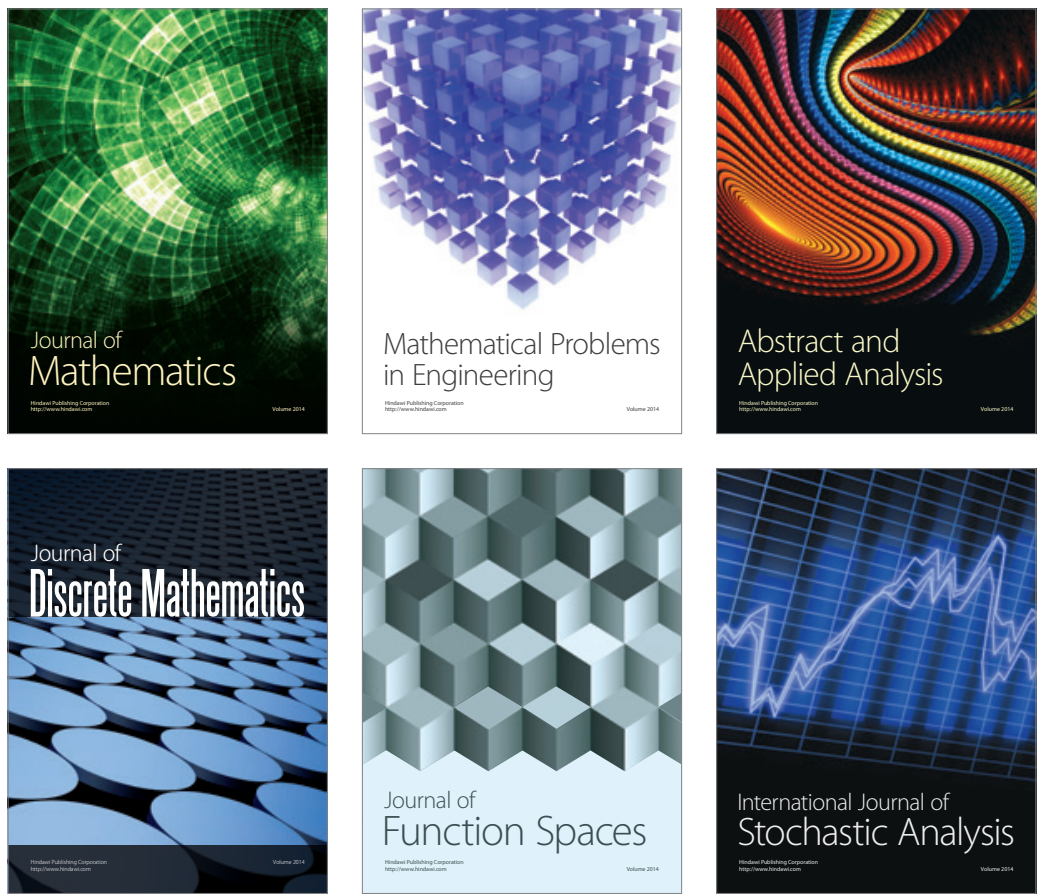

Journal of

Function Spaces

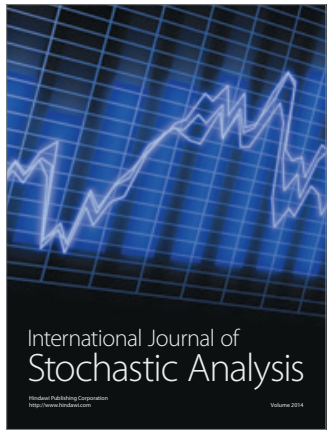

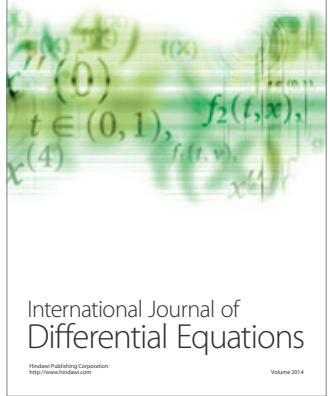
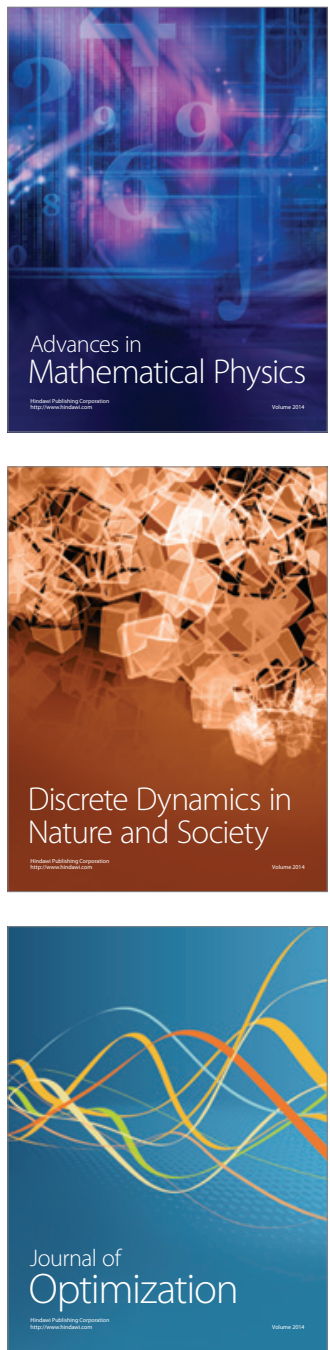